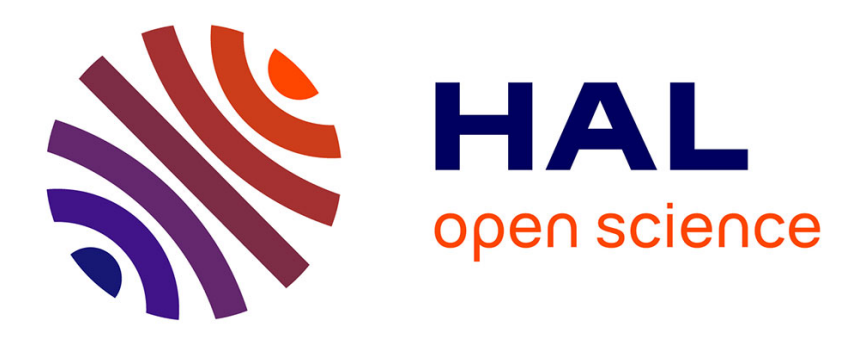

\title{
A Comprehensive Introduction to sub-Riemannian Geometry
}

Andrei Agrachev, Davide Barilari, Ugo Boscain

\section{To cite this version:}

Andrei Agrachev, Davide Barilari, Ugo Boscain. A Comprehensive Introduction to sub-Riemannian Geometry. Cambridge University Press, 2020, 978-1-108-47635-5. hal-02019181

\section{HAL Id: hal-02019181 \\ https://hal.science/hal-02019181}

Submitted on 28 Mar 2019

HAL is a multi-disciplinary open access archive for the deposit and dissemination of scientific research documents, whether they are published or not. The documents may come from teaching and research institutions in France or abroad, or from public or private research centers.
L'archive ouverte pluridisciplinaire HAL, est destinée au dépôt et à la diffusion de documents scientifiques de niveau recherche, publiés ou non, émanant des établissements d'enseignement et de recherche français ou étrangers, des laboratoires publics ou privés. 


\title{
A Comprehensive Introduction to sub-Riemannian Geometry
}

from Hamiltonian viewpoint

\author{
ANDREI AGRACHEV \\ DAVIDE BARILARI \\ UGO BOSCAIN
}

February 6, 2019

Draft version 


\section{Preface}

This book presents material taught by the authors in graduated courses at Trieste (SISSA), Paris (Institut Henri Poincaré, Orsay, Paris Diderot), and several summer schools, in the period 2008 2018.

It contains material for an introductory course in sub-Remannan geometry at master or $\mathrm{PhD}$ level, as well as material for a more advanced course.

The book attempts to be as elementary as possible but, although the main concepts are recalled, it requires a certain ability in managing object in differential geometry (vector fields, differential forms, symplectic manifolds, etc.). We try to avoid as much as possible the use of functional analysis (some is required starting from Chapter 6).

We do not require any knowledge in Riemannian geometry. Actually from the book one can extract an introductory course in Riemannian geometry as a special case of sub-Riemannian one, starting from the geometry of surfaces in Chapter 1.

There are few other books of sub-Riemannian geometry available. Besides the pioneering book edited by A. Bellaïche and J.-J. Risler [BR96], a nowadays classical reference is the book of R. Montgomery [Mon02], that inspired several of our chapters. More recent books, written in a language similar to the one we use, are those of F. Jean [Jea14] and L. Rifford [Rif14]; see also the collection of lectures notes [BBS16a, BBS16b]. Other related books, although with a different approach, are the monographs [BLU07] and [CDPT07].

Example of an introductory course of sub-Riemannan geometry. Chapters 2, 3 (without the appendices), 4, 7 (without 7.1), 9, 13, 21.

\section{Example of an advanced course of sub-Riemannan geometry.}

Chapters 2, 3 (with the appendices), 4, 6, 7 (together with 7.1), 8, 9, 10, 11, 12, 13, 14, 15, 17, 18, 19, 20, 21, the appendix by Zelenko.

\section{Example of a course of Riemannan geometry.}

Chapters 1, 2, 3 (without the appendices), 4, 5, 7, 8, 11, 14 (without 14.4-14.5-14.6), 15, 16, 21 (only 21.1).

Acknowledgments. A special thanks goes to Luca Rizzi and Mario Sigalotti, who contributed with several crucial corrections and suggestions on different topics of the book.

We are grateful to all the colleagues that helped us in the final reading of the manuscript, Ivan Beschastnyi, Francesco Boarotto, Daniele Cannarsa, Francesca Chittaro, Valentina Franceschi, Roberta Ghezzi, Frédéric Jean, Antonio Lerario, Paolo Mason, Eugenio Pozzoli, Dario Prandi, Ludovic Sacchelli, Yuri Sachkov, and Michele Stecconi. 
We are also grateful to the colleagues that during the years gave us suggestions to improve both the content and the exposition: Carolina Biolo, Andrea Bonfiglioli, Jean-Baptiste Caillau, JeanPaul Gauthier, Moussa Gaye, Velimir Jurdjevic, Andrea Mondino, Richard Montgomery, Roberto Monti, Elisa Paoli.

This project has been supported by the European Research Council: ERC StG 2009 "GeCoMethods", project number 239748 and ERC POC 2016 "ARTIV1", project number 727283. Also it was supported by the ANR project SRGI "Sub-Riemannian Geometry and Interactions", contract number ANR-15-CE40-0018. 


\section{Contents}

$\begin{array}{ll}\text { Introduction } & 12\end{array}$

1 Geometry of surfaces in $\mathbb{R}^{3} \quad 21$

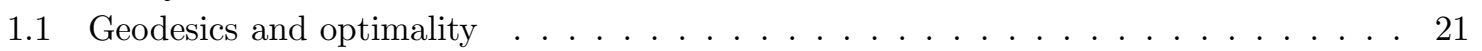

1.1.1 Existence and minimizing properties of geodesics . . . . . . . . . . . 25

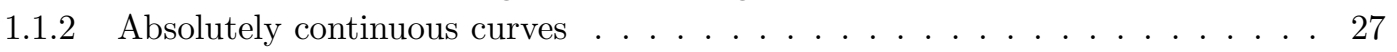

1.2 Parallel transport . . . . . . . . . . . . . . . . . . . . . . 27

1.2.1 Parallel transport and Levi-Civita connection . . . . . . . . . . . . . . . . 29

1.3 Gauss-Bonnet theorems ... . . . . . . . . . . . . . . . . . 31

1.3.1 Gauss-Bonnet theorem: local version . . . . . . . . . . . . . . . . 31

1.3.2 Gauss-Bonnet theorem: global version . . . . . . . . . . . . . . . 35

1.3.3 Consequences of the Gauss-Bonnet theorems . . . . . . . . . . . . . 38

1.3.4 The Gauss map . . . . . . . . . . . . . . . . . . . . 39

1.4 Surfaces in $\mathbb{R}^{3}$ with the Minkowski inner product . . . . . . . . . . . . . . . . . . 42

1.5 Model spaces of constant curvature . . . . . . . . . . . . . . . . . . . 45

1.5.1 Zero curvature: the Euclidean plane . . . . . . . . . . . . . . . 45

1.5.2 Positive curvature: the sphere . . . . . . . . . . . . . . . 45

1.5.3 Negative curvature: the hyperbolic plane . . . . . . . . . . . . . . . . . 47

1.6 Bibliographical note . . . . . . . . . . . . . . . . . . . . . 48

2 Vector fields $\quad 49$

2.1 Differential equations on smooth manifolds . . . . . . . . . . . . . . . . . . . 49

2.1 .1 Tangent vectors and vector fields . . . . . . . . . . . . . . . . 49

2.1 .2 Flow of a vector field . . . . . . . . . . . . . . . . . . 51

2.1.3 Vector fields as operators on functions . . . . . . . . . . . . . . . 51

2.1.4 Nonautonomous vector fields . . . . . . . . . . . . . . . . . . . . . 52

2.2 Differential of a smooth map . . . . . . . . . . . . . . . . . . . . . . . . . . 54

2.3 Lie brackets . . . . . . . . . . . . . . . . . . . . . . . . . 56

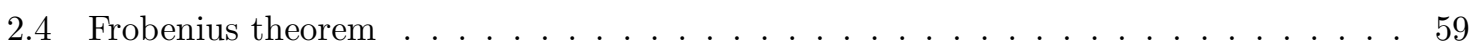

2.4.1 An application of Frobenius theorem . . . . . . . . . . . . . . 61

2.5 Cotangent space ............................ 62

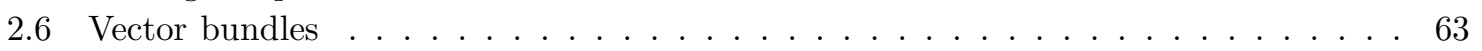

2.7 Submersions and level sets of smooth maps . . . . . . . . . . . . . . . . 65

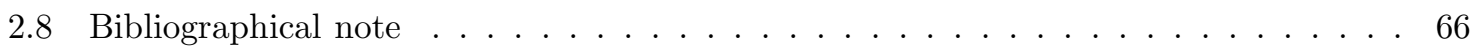


3 Sub-Riemannian structures $\quad \mathbf{6 7}$

3.1 Basic definitions . . . . . . . . . . . . . . . . . . . . . 67

3.1.1 The minimal control and the length of an admissible curve . . . . . . . . 70

3.1.2 Equivalence of sub-Riemannian structures . . . . . . . . . . . . . . 73

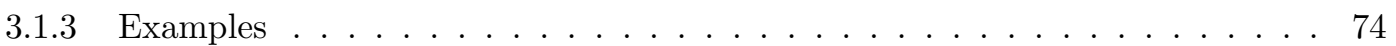

3.1.4 Every sub-Riemannian structure is equivalent to a free one . . . . . . . . . 76

3.2 Sub-Riemannian distance and Rashevskii-Chow theorem . . . . . . . . . . . . . . . . 77

3.2.1 Proof of Rashevskii-Chow theorem . . . . . . . . . . . . . . . 78

3.2 .2 Non bracket-generating structures . . . . . . . . . . . . . . . . 82

3.3 Existence of length-minimizers . . . . . . . . . . . . . . . . . . . . 83

3.3.1 On the completeness of the sub-Riemannian distance . . . . . . . . . . 85

3.3.2 Lipschitz curves with respect to $d$ vs admissible curves . . . . . . . . . . . . 87

3.3.3 Lipschitz equivalence of sub-Riemannian distances . . . . . . . . . . . . . . 88

3.3.4 Continuity of $d$ with respect to the sub-Riemannian structure . . . . . . . . . 89

3.4 Pontryagin extremals . . . . . . . . . . . . . . . . . . . . . . 91

3.4.1 The energy functional . . . . . . . . . . . . . . . . 93

3.4 .2 Proof of Theorem $3.59 \ldots \ldots \ldots \ldots$. . . . . . . . . . . . . . 94

3.5 Appendix: Measurability of the minimal control . . . . . . . . . . . . . . . . . 97

3.5.1 A measurability lemma . . . . . . . . . . . . . . . . . 97

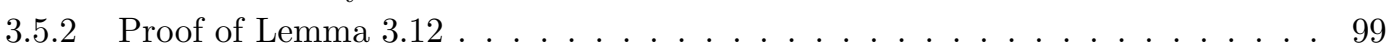

3.6 Appendix: Lipschitz vs absolutely continuous admissible curves . . . . . . . . . . . . 99

3.7 Bibliographical note ... . . . . . . . . . . . . . . . . 100

4 Pontryagin extremals: characterization and local minimality 101

4.1 Geometric characterization of Pontryagin extremals . . . . . . . . . . . . . . . . . . 101

4.1.1 Lifting a vector field from $M$ to $T^{*} M \ldots \ldots$. . . . . . . . . . . 102

4.1 .2 The Poisson bracket . . . . . . . . . . . . . . . . . . 103

4.1.3 Hamiltonian vector fields . . . . . . . . . . . . . . . . 105

4.2 The symplectic structure . . . . . . . . . . . . . . . . . . . . 107

4.2.1 Symplectic form vs Poisson bracket . . . . . . . . . . . . . . . 108

4.3 Characterization of normal and abnormal Pontryagin extremals . . . . . . . . . . . . 109

4.3.1 Normal extremals . . . . . . . . . . . . . . . . . . . . . . 110

4.3.2 Abnormal extremals . . . . . . . . . . . . . . . . . . 113

4.3.3 Codimension one and contact distributions . . . . . . . . . . . . . . . 115

4.4 Examples .............................. . . 116

4.4 2D Riemannian geometry . . . . . . . . . . . . . . . 116

4.4 .2 Isoperimetric problem . . . . . . . . . . . . . . . . . . . . . 119

4.4 .3 Heisenberg group . . . . . . . . . . . . . . . . . . . . 122

4.5 Lie derivative . . . . . . . . . . . . . . . . . . . . . . . . . . 123

4.6 Symplectic manifolds . . . . . . . . . . . . . . . . . . . . . . . . 125

4.7 Local minimality of normal extremal trajectories . . . . . . . . . . . . . . . . . . 127

4.7.1 The Poincaré-Cartan one-form . . . . . . . . . . . . . . . . 127

4.7.2 Normal Pontryagin extremal trajectories are geodesics . . . . . . . . . . . . 129

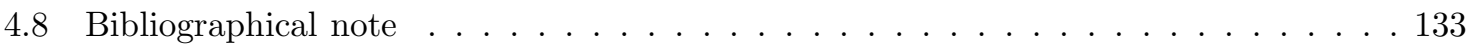


$5 \quad$ First integrals and integrable systems

5.1 Reduction of Hamiltonian systems with symmetries . . . . . . . . . . . . . 135

5.1.1 An example of symplectic reduction: the space of affine lines in $\mathbb{R}^{n} \ldots \ldots .137$

5.2 Riemannian geodesic flow on hypersurfaces . . . . . . . . . . . . . 138

5.2 .1 Geodesics on hypersurfaces . . . . . . . . . . . . . . . . . 138

5.2 .2 Riemannian geodesic flow and symplectic reduction . . . . . . . . . . 139

5.3 Sub-Riemannian structures with symmetries . . . . . . . . . . . . . . . 141

5.4 Completely integrable systems . . . . . . . . . . . . . . . . . . . . 143

5.5 Arnold-Liouville theorem . . . . . . . . . . . . . . . . . . . . . . . 146

5.6 Geodesic flows on quadrics . . . . . . . . . . . . . . . . . . . . . . . 148

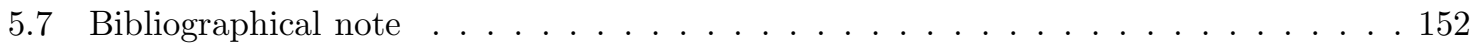

6 Chronological calculus $\quad \mathbf{1 5 3}$

6.1 Motivation . . . . . . . . . . . . . . . . . . . . . . 153

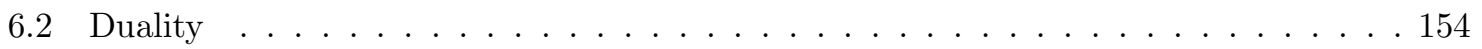

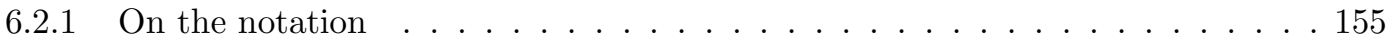

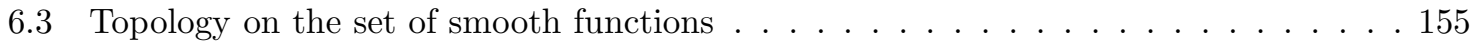

6.3 .1 Family of functionals and operators . . . . . . . . . . . . 156

6.4 Operator ODEs and Volterra expansions . . . . . . . . . . . . . . . . 157

6.4 .1 Volterra expansion . . . . . . . . . . . . . . . . . . 158

6.4 Adjoint representation . . . . . . . . . . . . . . . . . . . . 160

6.5 Variations formulae . . . . . . . . . . . . . . . . . . . . . 162

6.6 Appendix: Estimates and Volterra expansion . . . . . . . . . . . . . . . . . 163

6.7 Appendix: Remainder term of the Volterra expansion . . . . . . . . . . . . . 166

6.8 Bibliographical note . . . . . . . . . . . . . . . . . . . . . . 168

$7 \quad$ Lie groups and left-invariant sub-Riemannian structures $\quad 169$

7.1 Subgroups of $\operatorname{Diff}(M)$ generated by a finite-dimensional Lie algebra of vector fields . 169

7.1.1 A finite-dimensional approximation . . . . . . . . . . . . . . . 170

7.1 .2 Passage to infinite dimension . . . . . . . . . . . . . . . 173

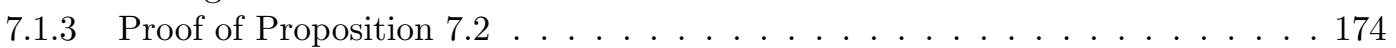

7.2 Lie groups and Lie algebras . . . . . . . . . . . . . . . . . . . . 175

7.2 .1 Lie groups as groups of diffeomorphisms . . . . . . . . . . . . . . 176

7.2 .2 Matrix Lie groups and the matrix notation . . . . . . . . . . . . . . . 178

7.2.3 Bi-invariant pseudo-metrics and Haar measures . . . . . . . . . . . . . . . . 180

7.2.4 The Levi-Malcev decomposition . . . . . . . . . . . . . . . . . . . . . . . . 182

7.3 Trivialization of $T G$ and $T^{*} G \ldots \ldots \ldots \ldots \ldots \ldots \ldots$

7.4 Left-invariant sub-Riemannian structures . . . . . . . . . . . . . . . 183

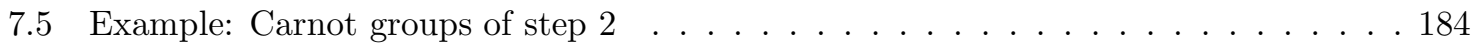

7.5.1 Normal Pontryagin extremals for Carnot groups of step $2 \ldots \ldots$. . . . . 186

7.6 Left-invariant Hamiltonian systems on Lie groups . . . . . . . . . . . . . . . . . 189

7.6.1 Vertical coordinates in $T G$ and $T^{*} G \ldots \ldots$. . . . . . . . . 189

7.6.2 Left-invariant Hamiltonians . . . . . . . . . . . . . . . . . . . . . . . 191

7.7 Normal extremals for left-invariant sub-Riemannian structures . . . . . . . . . . . 193

7.7.1 Explicit expression of normal Pontryagin extremals in the $\mathbf{d} \oplus \mathbf{s}$ case . . . . . 194

7.7.2 Example: The $\mathbf{d} \oplus \mathbf{s}$ problem on $S O(3) \ldots \ldots$. . . . . . . . . 195 
7.7.3 Further comments on the $\mathbf{d} \oplus$ s problem: $S O(3)$ and $S O_{+}(2,1) \ldots \ldots$

7.7.4 Explicit expression of normal Pontryagin extremals in the $\mathbf{k} \oplus \mathbf{z}$ case . . . . 199

7.8 Rolling spheres . . . . . . . . . . . . . . . . . . . . . . 202

7.8 .1 Rolling with twisting . . . . . . . . . . . . . . . . . . 202

7.8 .2 Rolling without twisting . . . . . . . . . . . . . . . . . 205

7.8.3 Euler's "cvrvae elasticae" . . . . . . . . . . . . . . . . . . . . . . . . . 210

7.8.4 Rolling spheres: further comments . . . . . . . . . . . . . . . . . . . 212

7.9 Bibliographical note . . . . . . . . . . . . . . . . . . . . . 213

8 End-point map and exponential map $\mathbf{2 1 5}$

8.1 The end-point map . . . . . . . . . . . . . . . . . . . . . . . 215

8.1.1 Regularity of the end-point map: proof of Proposition 8.5 . . . . . . . . 216

8.2 Lagrange multipliers rule . . . . . . . . . . . . . . . . . . . . . . . 219

8.3 Pontryagin extremals via Lagrange multipliers . . . . . . . . . . . . . . . . . . . . 219

8.4 Critical points and second order conditions . . . . . . . . . . . . . . 220

8.4.1 The manifold of Lagrange multipliers . . . . . . . . . . . . . . . . . . . 223

8.5 Sub-Riemannian case . . . . . . . . . . . . . . . . . . . . . 228

8.6 Exponential map and Gauss' Lemma . . . . . . . . . . . . . . . . . . . . . 231

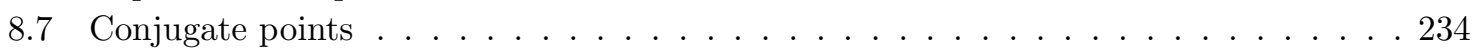

8.8 Minimizing properties of extremal trajectories . . . . . . . . . . . . . . 238

8.8.1 Local length-minimality in the $W^{1,2}$ topology. Proof of Theorem 8.52 . . . . 239

8.8.2 Local length-minimality in the $C^{0}$ topology . . . . . . . . . . . . . . . . 241

8.9 Compactness of length-minimizers . . . . . . . . . . . . . . . . . 245

8.10 Cut locus and global length-minimizers _ . . . . . . . . . . . . . 247

8.11 An example: the first conjugate locus on perturbed sphere . . . . . . . . . . . 250

8.12 Bibliographical note . . . . . . . . . . . . . . . . . . . . . 252

9 2D Almost-Riemannian Structures $\quad 255$

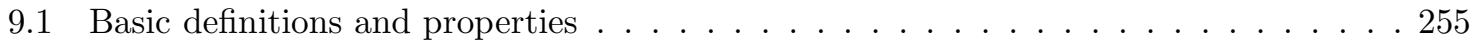

9.1.1 How big is the singular set? . . . . . . . . . . . . . . . . 260

9.1.2 Genuinely 2D almost-Riemannian structures have always infinite area . . . 261

9.1 .3 Pontryagin extremals . . . . . . . . . . . . . . . . . . . . 263

9.2 The Grushin plane . . . . . . . . . . . . . . . . . . . . . . . . . . . . . . . . 264

9.2.1 Geodesics on the Grushin plane . . . . . . . . . . . . . . . . . 265

9.3 Riemannian, Grushin and Martinet points . . . . . . . . . . . . . . . 266

9.3 .1 Normal forms . . . . . . . . . . . . . . . . . . . . . . . . . 270

9.4 Generic 2D almost-Riemannian structures . . . . . . . . . . . . . . . . 272

9.4.1 Proof of the genericity result _ . . . . . . . . . . . . . . . 272

9.5 A Gauss-Bonnet theorem . . . . . . . . . . . . . . . . . . . . . 273

9.5.1 Integration of the curvature . . . . . . . . . . . . . . 274

9.5 .2 The Euler number . . . . . . . . . . . . . . . . . . . . . 275

9.5.3 Gauss-Bonnet theorem . . . . . . . . . . . . . . . . . . . 276

9.5.4 Every compact orientable 2D manifold can be endowed with a free almost-

Riemannian structure with only Riemannian and Grushin points . . . . . . . 282

9.6 Bibliographical note . . . . . . . . . . . . . . . . . . . . . . . . . . 282 
10 Nonholonomic tangent space

10.1 Flag of the distribution and Carnot groups . . . . . . . . . . . . . . . 285

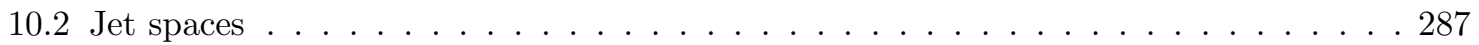

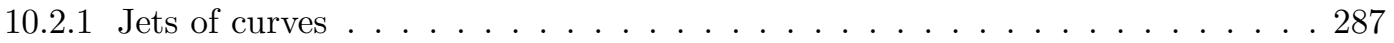

10.2 .2 Jets of vector fields . . . . . . . . . . . . . . . . . . . 289

10.3 Admissible variations and nonholonomic tangent space . . . . . . . . . . . . 290

10.3.1 Admissible variations . . . . . . . . . . . . . . . . . . . . 291

10.3.2 Nonholonomic tangent space . . . . . . . . . . . . . . . . . . 292

10.4 Nonholonomic tangent space and privileged coordinates _ . . . . . . . . . . . 294

10.4.1 Privileged coordinates . . . . . . . . . . . . . . . . . . 294

10.4.2 Description of the nonholonomic tangent space in privileged coordinates . . . 297

10.4.3 Existence of privileged coordinates: proof of Theorem 10.32 . . . . . . . . 303

10.4.4 Nonholonomic tangent spaces in low dimension . . . . . . . . . . . . 307

10.5 Metric meaning . . . . . . . . . . . . . . . . . . . . . . . . 309

10.5.1 Convergence of the sub-Riemannian distance and the Ball-Box theorem . . . 310

10.6 Algebraic meaning . . . . . . . . . . . . . . . . . . . . . 314

10.6.1 Nonholonomic tangent space: the equiregular case . . . . . . . . . . 316

10.7 Carnot groups: normal forms in low dimension . . . . . . . . . . . . . . 317

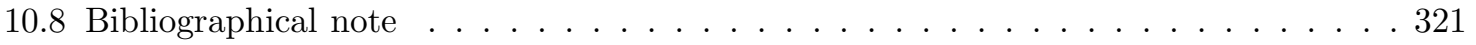

11 Regularity of the sub-Riemannian distance 323

11.1 Regularity of the sub-Riemannian squared distance . . . . . . . . . . . . . . . 323

11.2 Locally Lipschitz functions and maps . . . . . . . . . . . . . . . . . . . . . 330

11.2.1 Locally Lipschitz map and Lipschitz submanifolds . . . . . . . . . . . . . . 334

11.2.2 A non-smooth version of Sard Lemma . . . . . . . . . . . . . . . . . 336

11.3 Regularity of sub-Riemannian spheres . . . . . . . . . . . . . . . . . . 339

11.4 Geodesic completeness and Hopf-Rinow theorem . . . . . . . . . . . . . . . 342

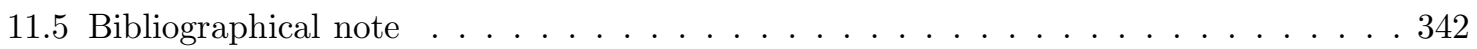

12 Abnormal extremals and second variation $\quad 345$

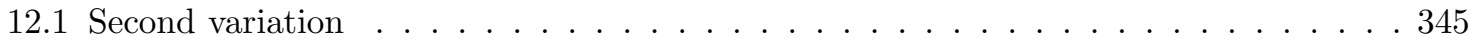

12.2 Abnormal extremals and regularity of the distance . . . . . . . . . . . 346

12.3 Goh and generalized Legendre conditions . . . . . . . . . . . . . . . . 352

12.3.1 Proof of Goh condition - (i) of Theorem 12.13 . . . . . . . . . . . . 354

12.3.2 Proof of generalized Legendre condition - (ii) of Theorem 12.13 . . . . . . 360

12.3.3 More on Goh and generalized Legendre conditions . . . . . . . . . . . . 361

12.4 Rank 2 distributions and nice abnormal extremals . . . . . . . . . . . . . 363

12.5 Minimality of nice abnormal in rank 2 structures $\ldots \ldots \ldots 66$

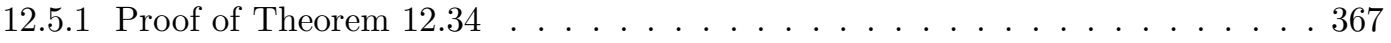

12.6 Conjugate points along abnormals $\ldots \ldots \ldots \ldots \ldots \ldots \ldots \ldots$

12.6.1 Abnormals in dimension $3 \ldots \ldots$. . . . . . . . . . . . . . . . . . . . . . . . . . . .

12.6.2 Higher dimension . . . . . . . . . . . . . . . . . . . . . . . . 380

12.7 Equivalence of local minimality with respect to $W^{1,2}$ and $C^{0}$ topology $\ldots \ldots . \ldots 381$

12.8 Non-minimality of corners . . . . . . . . . . . . . . . . . . 383

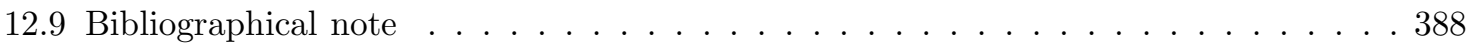


13.1 Carnot groups of step 2 . . . . . . . . . . . . . . . . . . . . . . . . 392

13.2 Multi-dimensional Heisenberg groups . . . . . . . . . . . . . . . . . . . . 394

13.2.1 Pontryagin extremals in the contact case . . . . . . . . . . . . . . . . 395

13.2.2 Optimal synthesis . . . . . . . . . . . . . . . . . . . 397

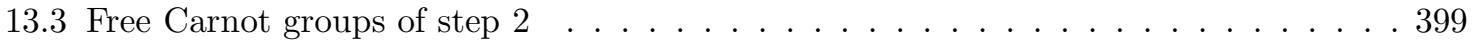

13.3.1 Intersection of the cut locus with the vertical subspace . . . . . . . . . . . 402

13.3.2 The cut locus for the free step-two Carnot group of rank three . . . . . . . . 403

13.4 An extended Hadamard technique to compute the cut locus . . . . . . . . . . . . . 404

13.5 The Grushin structure . . . . . . . . . . . . . . . . . . . . . . . . . . . 409

13.5.1 Optimal synthesis starting from a Riemannian point . . . . . . . . . . . 409

13.5.2 Optimal synthesis starting from a singular point . . . . . . . . . . . . . . . . . . . . . . . . . . . . .

13.6 The standard sub-Riemannian structure on $S U(2)$. . . . . . . . . . . . . . . . . . . 415

13.7 Optimal synthesis on the groups $S O(3)$ and $S O_{+}(2,1) \ldots \ldots$. . . . . . . . . . . . . . 419

13.8 Synthesis for the group of Euclidean transformations of the plane $S E(2)$. . . . . . 423 13.8.1 Mechanical interpretation . . . . . . . . . . . . . . . . . . . . . . . . . . . . . . . . . . . . 424

13.8 .2 Geodesics . . . . . . . . . . . . . . . . . . . . . . . . . . . . . . . . . . . . . . . . .

13.9 The Martinet flat sub-Riemannian structure . . . . . . . . . . . . . . . . . . . . 431

13.9.1 Abnormal extremals . . . . . . . . . . . . . . . . . . 431

13.9.2 Normal extremals ... . . . . . . . . . . . . . . . . . . . . . . . . . . . . . . . . . . . . . . . . . .

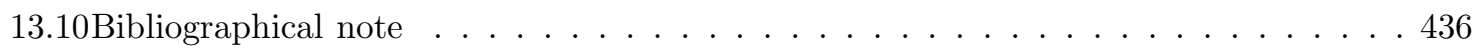

14 Curves in the Lagrange Grassmannian $\quad 441$

14.1 The geometry of the Lagrange Grassmannian . . . . . . . . . . . . . . . . . . . . . . 441

14.1.1 The Lagrange Grassmannian . . . . . . . . . . . . . . . . . . . . . . . . . . 444

14.2 Regular curves in Lagrange Grassmannian . . . . . . . . . . . . . . . . . . . . . . . . 446

14.3 Curvature of a regular curve . . . . . . . . . . . . . . . . . . . . . . . . 449

14.4 Reduction of non-regular curves in Lagrange Grassmannian . . . . . . . . . . . . . . 452

14.5 Ample curves . . . . . . . . . . . . . . . . . . . . . . . . . . . . 453

14.6 From ample to regular . . . . . . . . . . . . . . . . . . . . . . 455

14.7 Conjugate points in $L(\Sigma)$. . . . . . . . . . . . . . . . . . . . . . . . . . . . . . . 459

14.8 Comparison theorems for regular curves . . . . . . . . . . . . . . . . . . . . . 460

14.9 Bibliographical note ... . . . . . . . . . . . . . . . . . 463

15 Jacobi curves $\quad 465$

15.1 From Jacobi fields to Jacobi curves . . . . . . . . . . . . . . . . . . . . . . 465

15.1 .1 Jacobi curves . . . . . . . . . . . . . . . . . . . . . . . . . . . . . . . . . . . . . . . . . . . . . . . . . . . . .

15.2 Conjugate points and optimality . . . . . . . . . . . . . . . . . 468

15.3 Reduction of the Jacobi curves by homogeneity . . . . . . . . . . . . . . . . . . 469

15.4 Bibliographical note ... . . . . . . . . . . . . . . . . . . . 471

16 Riemannian curvature $\quad 473$

16.1 Ehresmann connection . . . . . . . . . . . . . . . . . . . . 473

16.1.1 Curvature of an Ehresmann connection . . . . . . . . . . . . . . . . . 474

16.1.2 Linear Ehresmann connections . . . . . . . . . . . . . . . . . . 475

16.1.3 Covariant derivative and torsion for linear connections . . . . . . . . . . . 476 
16.2 Riemannian connection . . . . . . . . . . . . . . . . . . 478

16.3 Relation with Hamiltonian curvature . . . . . . . . . . . . . . . . . . . . 483

16.4 Comparison theorems for conjugate points . . . . . . . . . . . . . . . 485

16.5 Locally flat spaces . . . . . . . . . . . . . . . . . . . . . 486

16.6 Curvature of 2D Riemannian manifolds _ . . . . . . . . . . . . . . . . 487

16.7 Bibliographical note . . . . . . . . . . . . . . . . . . . 488

17 Curvature in 3D contact sub-Riemannian geometry 489

17.1 A worked-out example: the 2D Riemannian case . . . . . . . . . . . . . . . 489

17.2 3D contact sub-Riemannian manifolds . . . . . . . . . . . . . . . 493

17.3 Canonical frames . . . . . . . . . . . . . . . . . . . . 496

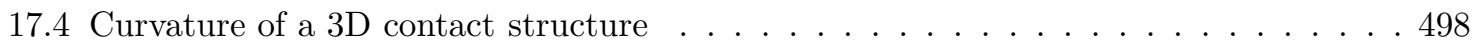

17.4.1 Geometric interpretation . . . . . . . . . . . . . . . . . . . 503

17.5 Local classification of 3D left-invariant structures . . . . . . . . . . . . . . . 504

17.5.1 A description of the classification . . . . . . . . . . . . . . 506

17.5.2 A sub-Riemannian isometry between non isomorphic Lie groups . . . . . . 508

17.5.3 Canonical frames and classification. Proof of Theorem 17.29 . . . . . . . . 510

17.5.4 An explicit isometry. Proof of Theorem 17.32 . . . . . . . . . . . . . 513

17.6 Appendix: Remarks on curvature coefficients . . . . . . . . . . . . . . . . 517

17.7 Bibliographical note . . . . . . . . . . . . . . . . . . . . . . 518

18 Integrability of the sub-Riemannian geodesic flow on 3D Lie groups $\quad 519$

18.1 Poisson manifolds and symplectic leaves . . . . . . . . . . . . . . . . 519

18.2 Integrability of Hamiltonian systems on Lie groups . . . . . . . . . . . . . . . . 523

18.2.1 The Poisson manifold $\mathfrak{g}^{*} \ldots \ldots \ldots$. . . . . . . . . . . . . . . . 523

18.2.2 The Casimir first integral . . . . . . . . . . . . . . . . . . . . . 524

18.2.3 First integrals associated with a right-invariant vector field . . . . . . . . . 525

18.2.4 Complete integrability on Lie groups . . . . . . . . . . . . . . . . 526

18.3 Left-invariant Hamiltonian systems on 3D Lie groups . . . . . . . . . . . . . . . 526

18.3.1 Rank 2 sub-Riemannian structures on 3D Lie groups . . . . . . . . . . . . 530

18.3.2 Classification of symplectic leaves on 3D Lie groups . . . . . . . . . . . . . 532

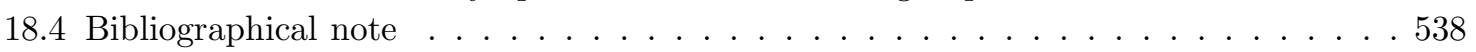

19 Asymptotic expansion of the 3D contact exponential map 541

19.1 The exponential map . . . . . . . . . . . . . . . . . . . . . . 541

19.1.1 The nilpotent case . . . . . . . . . . . . . . . . . . . . . . . 542

19.2 General case: second order asymptotic expansion . . . . . . . . . . . . . . . . . 544

19.2.1 Proof of Proposition 19.2: second order asymptotics . . . . . . . . . . . . 544

19.3 General case: higher order asymptotic expansion . . . . . . . . . . . . . . . 548

19.3.1 Proof of Theorem 19.6: asymptotics of the exponential map . . . . . . . . . 550

19.3.2 Asymptotics of the conjugate locus . . . . . . . . . . . . . . . . . . 553

19.3.3 Asymptotics of the conjugate length . . . . . . . . . . . . . 555

19.3.4 Stability of the conjugate locus . . . . . . . . . . . . . . 556

19.4 Bibliographical note . . . . . . . . . . . . . . . . . . . 557 
20 The volume in sub-Riemannian geometry $\quad 559$

20.1 Equiregular sub-Riemannian manifolds . . . . . . . . . . . . . . . . . . . . 559

20.2 The Popp volume . . . . . . . . . . . . . . . . . . . . . . 5560

20.3 A formula for Popp volume in terms of adapted frames . . . . . . . . . . . . . 562

20.4 Popp volume and smooth isometries . . . . . . . . . . . . . . . . 565

20.5 Hausdorff dimension and Hausdorff volume . . . . . . . . . . . . . . 566

20.6 Hausdorff volume on sub-Riemannian manifolds . . . . . . . . . . . . . . . . 567

20.6 .1 Hausdorff dimension . . . . . . . . . . . . . . . . . . . . 568

20.6.2 On the metric convergence . . . . . . . . . . . . . . . . . . 570

20.6.3 Induced volumes and estimates . . . . . . . . . . . . . . . . 571

20.7 Density of the spherical Hausdorff volume with respect to a smooth volume . . . . . 573

20.8 Bibliographical note . . . . . . . . . . . . . . . . . . . . . . 574

21 The sub-Riemannian heat equation $\mathbf{5 7 5}$

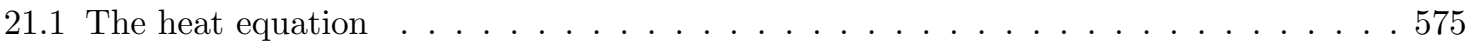

21.1.1 The heat equation in the Riemannian context . . . . . . . . . . . 575

21.1.2 The heat equation in the sub-Riemannian context . . . . . . . . . . . 578

21.1.3 The Hörmander theorem and the existence of the heat kernel . . . . . . . . . 580

21.1.4 The heat equation in the non bracket-generating case . . . . . . . . . . . 582

21.2 The heat-kernel on the Heisenberg group . . . . . . . . . . . . . . . . . . . . 582

21.2.1 The Heisenberg group as a group of matrices . . . . . . . . . . . . . . . . 583

21.2.2 The heat equation on the Heisenberg group . . . . . . . . . . . . . . . . 584

21.2.3 Construction of the Gaveau-Hulanicki fundamental solution . . . . . . . . . . 585

21.2.4 Small-time asymptotics for the Gaveau-Hulanicki fundamental solution . . . 592

21.3 Bibliographical note . . . . . . . . . . . . . . . . . . . . . . . 593

A Geometry of parametrized curves in Lagrangian Grassmannians (by Igor Zelenko)

A.1 Preliminaries . . . . . . . . . . . . . . . . . . . . . . 5595

A.2 Algebraic theory of curves in Grassmannians and flag varieties . . . . . . . . . . 601

A.3 Application to differential geometry of monotonic parametrized curves in Lagrangian

Grassmannians . . . . . . . . . . . . . . . . . . . . . 611

$\begin{array}{ll}\text { Bibliography } & 620\end{array}$

$\begin{array}{ll}\text { Index } & 637\end{array}$ 


\section{Introduction}

This book concerns a fresh development of the eternal idea of the distance as the length of a shortest path. In Euclidean geometry, shortest paths are segments of straight lines that satisfy all classical axioms. In the Riemannian world, Euclidean geometry is just one of a huge amount of possibilities. However, each of these possibilities is well approximated by Euclidean geometry at very small scale. In other words, Euclidean geometry is treated as geometry of initial velocities of the paths starting from a fixed point of the Riemannian space rather than the geometry of the space itself.

The Riemannian construction was based on the previous study of smooth surfaces in the Euclidean space undertaken by Gauss. The distance between two points on the surface is the length of a shortest path on the surface connecting the points. Initial velocities of smooth curves starting from a fixed point on the surface form a tangent plane to the surface, that is an Euclidean plane. Tangent planes at two different points are isometric, but neighborhoods of the points on the surface are not locally isometric in general; certainly not if the Gaussian curvature of the surface is different at the two points.

Riemann generalized Gauss' construction to higher dimensions and realized that it can be done in an intrinsic way; you do not need an ambient Euclidean space to measure the length of curves. Indeed, to measure the length of a curve it is sufficient to know the Euclidean length of its velocities. A Riemannian space is a smooth manifold whose tangent spaces are endowed with Euclidean structures; each tangent space is equipped with its own Euclidean structure that smoothly depends on the point where the tangent space is attached.

For a habitant sitting at a point of the Riemannian space, tangent vectors give directions where to move or, more generally, to send and receive information. He measures lengths of vectors, and angles between vectors attached at the same point, according to the Euclidean rules, and this is essentially all what he can do. It is important that our habitant can, in principle, completely recover the geometry of the space by performing these simple measurements along different curves.

In the sub-Riemannian space we cannot move, receive and send information in all directions. There are restrictions (imposed by the God, the moral imperative, the government, or simply a physical law). A sub-Riemannian space is a smooth manifold with a fixed admissible subspace in any tangent space where admissible subspaces are equipped with Euclidean structures. Admissible paths are those curves whose velocities are admissible. The distance between two points is the infimum of the length of admissible paths connecting the points. It is assumed that any pair of points in the same connected component of the manifold can be connected by at least an admissible path. The last assumption might look strange at a first glance, but it is not. The admissible subspace depends on the point where it is attached, and our assumption is satisfied for a more or less general smooth dependence on the point; better to say that it is not satisfied only for very special families of admissible subspaces.

Let us describe a simple model. Let our manifold be $\mathbb{R}^{3}$ with coordinates $x, y, z$. We consider 
the differential 1-form $\omega=-d z+\frac{1}{2}(x d y-y d x)$. Then $d \omega=d x \wedge d y$ is the pullback on $\mathbb{R}^{3}$ of the area form on the $x y$-plane. In this model the subspace of admissible velocities at the point $(x, y, z)$ is assumed to be the kernel of the form $\omega$. In other words, a curve $t \mapsto(x(t), y(t), z(t))$ is an admissible path if and only if $\dot{z}(t)=\frac{1}{2}(x(t) \dot{y}(t)-y(t) \dot{x}(t))$ or equivalently if

$$
z(t)=z(0)+\frac{1}{2} \int_{0}^{t}(x(s) \dot{y}(s)-y(s) \dot{x}(s)) d s .
$$

If $x(0)=y(0)=z(0)=0$, then $z(t)$ is the signed area of the domain bounded by the curve and the segment connecting $(0,0)$ with $(x(t), y(t))$.

In this geometry, the length of an admissible tangent vector $(\dot{x}, \dot{y}, \dot{z})$ is defined to be $\left(\dot{x}^{2}+\dot{y}^{2}\right)^{\frac{1}{2}}$, that is the length of the projection of the vector to the $x y$-plane. By construction, the subRiemannian length of the admissible curve in $\mathbb{R}^{3}$ is equal to the Euclidean length of its projection to the plane.

In this geometry, to compute the shortest paths connecting the origin $(0,0,0)$ to a fixed point $\left(x_{1}, y_{1}, z_{1}\right)$ we are then reduced to solve the classical Dido isoperimetric problem: find a shortest planar curve among those connecting $(0,0)$ with $\left(x_{1}, y_{1}\right)$ and such that the signed area of the domain bounded by the curve and the segment joining $(0,0)$ and $\left(x_{1}, y_{1}\right)$ is equal to $z_{1}$ (see Figure 1 ).

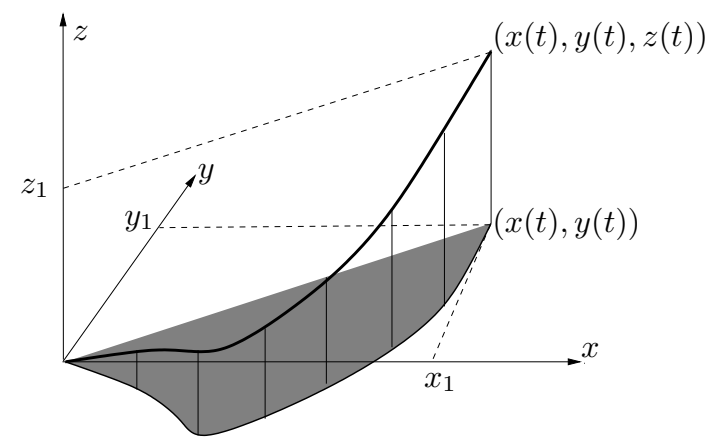

Figure 1: The Dido problem

Solutions of the Dido problem are arcs of circles and their lifts to $\mathbb{R}^{3}$ are spirals where $z(t)$ is the area of the piece of disc cut by the hord connecting $(0,0)$ with $(x(t), y(t))$ (see Figure 2).

A piece of such a spiral is a shortest admissible path between its endpoints while the planar projection of this piece is an arc of the circle. The spiral ceases to be a shortest path when its planar projection starts to run the circle for the second time, i.e., when the spiral starts its second turn. Sub-Riemannian balls centered at the origin for this model look like apples with singularities at the poles (see Figure 3).

Singularities are points on the sphere connected with the center by more than one shortest path. The dilation $(x, y, z) \mapsto\left(r x, r y, r^{2} z\right)$ transforms the ball of radius 1 into the ball of radius $r$. In particular, arbitrary small balls have singularities. This is always the case when admissible subspaces are proper subspaces.

Another important symmetry connects balls with different centers. Indeed, the product operation

$$
(x, y, z) \cdot\left(x^{\prime}, y^{\prime}, z^{\prime}\right) \doteq\left(x+x^{\prime}, y+y^{\prime}, z+z^{\prime}+\frac{1}{2}\left(x y^{\prime}-x^{\prime} y\right)\right)
$$




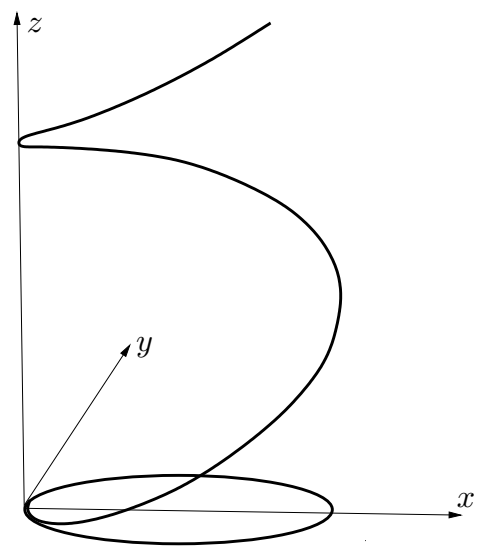

Figure 2: Solutions to the Dido problem

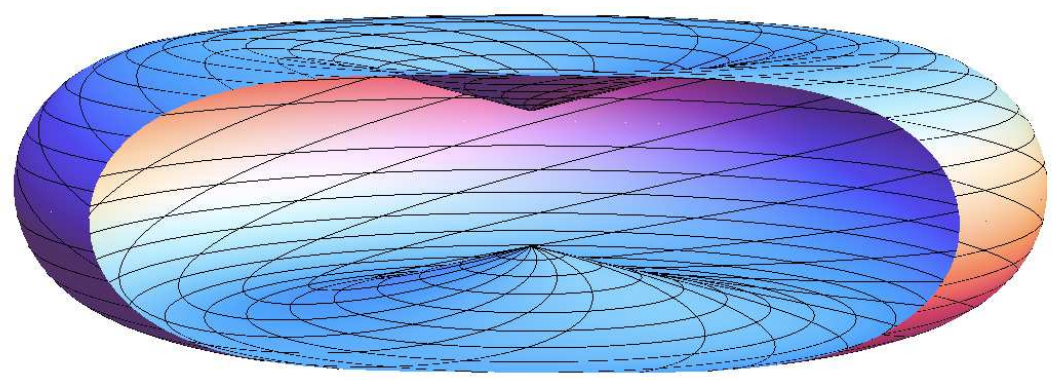

Figure 3: The Heisenberg sub-Riemannian sphere

turns $\mathbb{R}^{3}$ into a group, the Heisenberg group. The origin in $\mathbb{R}^{3}$ is the unit element of this group. It is easy to see that left-translations of the group transform admissible curves into admissible ones and preserve the sub-Riemannian length. Hence left translations transform balls in balls of the same radius. A detailed description of this example and other models of sub-Riemannian spaces is done in Sections 4.4.3, 7.5.1, 13.2.

Actually, even this simplest model tells us something about life in a sub-Riemannian space. Here we deal with planar curves but, in fact, operate in the three-dimensional space. Sub-Riemannian spaces always have a kind of hidden extra dimension. A good and not yet exploited source for mystic speculations but also for theoretical physicists who are always searching new crazy formalizations. In mechanics, this is a natural geometry for systems with nonholonomic constraints like skates, wheels, rolling balls, bearings etc. This kind of geometry could also serve to model social behavior that allows to increase the level of freedom without violation of a restrictive legal system.

Anyway, in this book we perform a purely mathematical study of sub-Riemannian spaces to provide an appropriate formalization ready for all potential applications. Riemannian spaces appear as a very special case. Of course, we are not the first to study the sub-Riemannian stuff. There is a broad literature even if there are not so many experts who could claim that sub-Riemannian geometry is his main field of expertise. Important motivations come from CR geometry, hyperbolic 
geometry, analysis of hypoelliptic operators, and some other domains. Our first motivation was control theory: length minimizing is a nice class of optimal control problems.

Indeed, one can find a control theory spirit in our treatment of the subject. First of all, we include admissible paths in admissible flows that are flows generated by vector fields whose values in all points belong to admissible subspaces. The passage from admissible subspaces attached at different points of the manifold to a globally defined space of admissible vector fields makes the structure more flexible and well-adapted to algebraic manipulations. We pick generators $f_{1}, \ldots, f_{k}$ of the space of admissible fields, and this allows us to describe all admissible paths as solutions to time-varying ordinary differential equations of the form: $\dot{q}(t)=\sum_{i=1}^{k} u_{i}(t) f_{i}(q(t))$. Different admissible paths correspond to the choice of different control functions $u_{i}(\cdot)$ and initial points $q(0)$ while the vector fields $f_{i}$ are fixed at the very beginning.

We also use a Hamiltonian approach supported by the Pontryagin maximum principle to characterize shortest paths. Few words about the Hamiltonian approach: sub-Riemannian geodesics are admissible paths whose sufficiently small pieces are length-minimizers, i.e. the length of such a piece is equal to the distance between its endpoints. In the Riemannian setting, any geodesic is uniquely determined by its velocity at the initial point $q$. In the general sub-Riemannian situation we have much more geodesics based at the the point $q$ than admissible velocities at $q$. Indeed, every point in a neighborhood of $q$ can be connected with $q$ by a length-minimizer, while the dimension of the admissible velocities subspace at $q$ is usually smaller than the dimension of the manifold.

What is a natural parametrization of the space of geodesics? To understand this question, we adapt a classical "trajectory - wave front" duality. Given a length-parameterized geodesic $t \mapsto \gamma(t)$, we expect that the values at a fixed time $t$ of geodesics starting at $\gamma(0)$ and close to $\gamma$ fill a piece of a smooth hypersurface (see Figure 4). For small $t$ this hypersurface is a piece of the sphere of radius $t$, while in general it is only a piece of the "wave front".

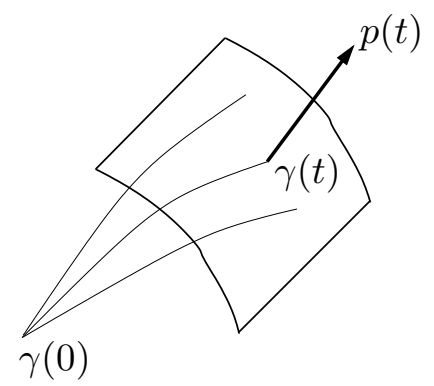

Figure 4: The "wave front" and the "impulse"

Moreover, we expect that $\dot{\gamma}(t)$ is transversal to this hypersurface. It is not always the case but this is true for a generic geodesic.

The "impulse" $p(t) \in T_{\gamma(t)}^{*} M$ is the covector orthogonal to the "wave front" and normalized by the condition $\langle p(t), \dot{\gamma}(t)\rangle=1$. The curve $t \mapsto(p(t), \gamma(t))$ in the cotangent bundle $T^{*} M$ satisfies a Hamiltonian system. This is exactly what happens in rational mechanics or geometric optics.

The sub-Riemannian Hamiltonian $H: T^{*} M \rightarrow \mathbb{R}$ is defined by the formula $H(p, q)=\frac{1}{2}\langle p, v\rangle^{2}$, where $p \in T_{q}^{*} M$, and $v \in T_{q} M$ is an admissible velocity of length 1 that maximizes $\langle p, w\rangle$ among all admissible velocities $w$ of length one at $q \in M$.

Any smooth function on the cotangent bundle defines a Hamiltonian vector field and such a 
field generates a Hamiltonian flow. The Hamiltonian flow on $T^{*} M$ associated to $H$ is the $s u b$ Riemannian geodesic flow. The Riemannian geodesic flow is just a special case.

As we mentioned, in general, the construction described above cannot be applied to all geodesics: the so-called abnormal geodesics are missed. An abnormal geodesic $\gamma(t)$ also possesses its "impulse" $p(t) \in T_{\gamma(t)}^{*} M$ but this impulse belongs to the orthogonal complement to the subspace of admissible velocities and does not satisfy the above Hamiltonian system. Geodesics that are trajectories of the geodesic flow are called normal. Actually, abnormal geodesics belong to the closure of the space of the normal ones, and elementary symplectic geometry provides a uniform characterization of the impulses for both classes of geodesics. Such a characterization is, in fact, a very special case of the Pontryagin maximum principle.

Recall that all velocities are admissible in the Riemannian case, and the Euclidean structure on the tangent bundle induces the identification of tangent vectors and covectors, i.e., of the velocities and impulses. We should however remember that this identification depends on the metric. One can think to a sub-Riemannian metric as the limit of a family of Riemannian metrics when the length of forbidden velocities tends to infinity, while the length of admissible velocities remains untouched. It is easy to see that the Riemannian Hamiltonians defined by such a family converge with all derivatives to the sub-Riemannian Hamiltonian. Hence the Riemannian geodesics with a prescribed initial impulse converge to the sub-Riemannian geodesic with the same initial impulse. On the other hand, we cannot expect any reasonable convergence for the family of Riemannian geodesics with a prescribed initial velocity: those with forbidden initial velocities disappear at the limit, while the number of geodesics with admissible initial velocities jumps to infinity.

\section{Outline of the book}

We start in Chapter 1 from surfaces in $\mathbb{R}^{3}$ that is the beginning of everything in differential geometry, and also a starting point of the story told in this book. There are not yet Hamiltonians here, but a control flavor is already present. The presentation is elementary and self-contained. A student in applied mathematics or analysis who missed the geometry of surfaces at the university or simply is not satisfied by his understanding of these classical ideas, might find it useful to read just this chapter even if he does not plan to study the rest of the book.

In Chapter 2, we recall some basic properties of vector fields and vector bundles. Sub-Riemannian structures are defined in Chapter 3 where we also study three fundamental facts: the finiteness and the continuity of the sub-Riemannian distance, the existence of length-minimizers, and the infinitesimal characterization of geodesics. The first is the classical Rashevskii-Chow theorem, the second and the third one are simplified versions of the Filippov existence theorem and of the Pontryagin maximum principle.

In Chapter 4, we introduce the symplectic language. We define the geodesic Hamiltonian flow, we consider some interesting two- and three-dimensional problems, and we prove a general sufficient condition for length-minimality of normal trajectories. Chapter 5 is devoted to integrable Hamiltonian systems. We explain the construction of the action-angle coordinates and we describe classical examples of integrable geodesic flows, such as the geodesic flow on ellipsoids.

Chapters 1-5 form a first part of the book where we do not use any tool from functional analysis. In fact, even the knowledge of the Lebesgue integration and elementary real analysis are not essential with a unique exception of the existence theorem in Section 3.3. In all other parts of the text, the reader will nevertheless understand the content just replacing the terms "Lipschitz" and "absolutely continuous" with "piecewise $C^{1}$ " and the term "measurable" with "piecewise continuous". 
We start to use some basic functional analysis in Chapter 6. In this chapter, we give elements of an operator calculus that simplifies and clarifies calculations with non-stationary flows, their variations and compositions. In Chapter 7 , we give a brief introduction to the Lie group theory. Lie groups are introduced as subgroups of the groups of diffeomorphisms of a manifold $M$ induced by a family of vector fields whose Lie algebra is finite dimensional. Then we study left-invariant sub-Riemannian structures and their geodesics.

In Chapter 8, we interpret the "impulses" as Lagrange multipliers for constrained optimization problems and apply this point of view to the sub-Riemannian case. We also introduce the subRiemannian exponential map and we study cut and conjugate points.

In Chapter 9, we consider two-dimensional sub-Riemannian metrics; such a metric coincides with a Riemannian one on an open and dense subset. We describe in details the model space of this geometry, known as the Grushin plane, and we discuss several properties in the generic case, among which a Gauss-Bonnet like theorem.

In Chapter 10, we construct the nonholonomic tangent space at a point $q$ of the manifold: a first quasi-homogeneous approximation of the space if you observe and exploit it from $q$ by means of admissible paths. In general, such a tangent space is a homogeneous space of a nilpotent Lie group equipped with an invariant vector distribution; its structure may depend on the point where the tangent space is attached. At generic points, this is a nilpotent Lie group endowed with a left-invariant vector distribution. The construction of the nonholonomic tangent space does not need a metric; if we take into account the metric, we obtain the Gromov-Hausdorff tangent to the sub-Riemannian metric space. Useful "ball-box" estimates of small balls follow automatically.

In Chapter 11, we study general analytic properties of the sub-Riemannian distance as a function of points of the manifold. It is shown that the distance is smooth on an open dense subset and is Lipschitz out of the points connected by abnormal length-minimizers. Moreover, if these bad points are absent, then almost every sphere is a Lipschitz submanifold.

In Chapter 12, we turn to abnormal geodesics, which provide the deepest singularities of the distance. Abnormal geodesics are critical points of the endpoint map defined on the space of admissible paths, and the main tool for their study is the Hessian of the endpoint map. This study permits to prove also that the cut locus from a point is adjacent to the point itself as soon as the structure is not Riemannian.

Chapter 13 is devoted to the explicit calculation of the sub-Riemannian optimal synthesis for model spaces. After a discussion on Carnot groups, we describe a technique based on the Hadamard theorem that permits, under certain assumptions, to compute the cut locus explicitly. We then apply this technique to several relevant examples.

This is the end of the second part of the book; next few chapters are devoted to the curvature and its applications. Let $\Phi^{t}: T^{*} M \rightarrow T^{*} M$, for $t \in \mathbb{R}$, be a sub-Riemannian geodesic flow. Submanifolds $\Phi^{t}\left(T_{q}^{*} M\right), q \in M$, form a fibration of $T^{*} M$. Given $\lambda \in T^{*} M$, let $J_{\lambda}(t) \subset T_{\lambda}\left(T^{*} M\right)$ be the tangent space to the leaf of this fibration.

Recall that $\Phi^{t}$ is a Hamiltonian flow and $T_{q}^{*} M$ are Lagrangian submanifolds; hence the leaves of our fibrations are Lagrangian submanifolds and $J_{\lambda}(t)$ is a Lagrangian subspace of the symplectic space $T_{\lambda}\left(T^{*} M\right)$.

In other words, $J_{\lambda}(t)$ belongs to the Lagrangian Grassmannian of $T_{\lambda}\left(T^{*} M\right)$, and $t \mapsto J_{\lambda}(t)$ is a curve in the Lagrangian Grassmannian, a Jacobi curve of the sub-Riemannian structure. The curvature of the sub-Riemannian space at $\lambda$ is simply the "curvature" of this curve in the Lagrangian Grassmannian. 
Chapter 14 is devoted to the elementary differential geometry of curves in the Lagrangian Grassmannian. In Chapter 15 we apply this geometry to Jacobi curves, that are curves in the Lagrange Grassmannian representing Jacobi fields.

The language of Jacobi curves is translated to the traditional language in the Riemannian case in Chapter 16. We recover the Levi-Civita connection and the Riemannian curvature and demonstrate their symplectic meaning. In Chapter 17, we explicitly compute the sub-Riemannian curvature for contact three-dimensional spaces and we show how the curvature invariants appear in the classification of sub-Riemannian left-invariant structures on 3D Lie groups. In Chapter 18, after a brief introduction on Poisson manifolds, we prove the integrability of the sub-Riemannian geodesic flow on 3D Lie groups. As a byproduct, we obtain a classification of coadjoint orbits on 3D Lie algebras. In the next Chapter 19 we study the small distance asymptotics of the exponential map for three-dimensional contact case and see how the structure of the conjugate locus is encoded in the curvature.

In Chapter 20 we address the problem of defining a canonical volume in sub-Riemannian geometry. First we introduce the Popp volume, that is a canonical volume that is smooth for equiregular sub-Riemannian manifold, and we study its basic properties. Then we define the Hausdorff volume and we study its density with respect to Popp's one.

In the last Chapter 21 we define the sub-Riemannian Laplace operator, and we study its properties (hypoellipticity, self-adjointness, etc.). We conclude with a discussion of the sub-Riemannian heat equation and an explicit formula for the heat kernel in the three-dimensional Heisenberg case.

The book is finished by an Appendix on the canonical frames for a wide class of curves in the Lagrangian Grassmannians, written by Igor Zelenko. This is a necessary background for a deeper systematic study of the curvature-type sub-Riemannian invariants, beyond the scope of this book.

We stop here this introduction into the "Comprehensive Introduction". We hope that the reader won't be bored; comments to the chapters contain references and suggestions for further reading. 